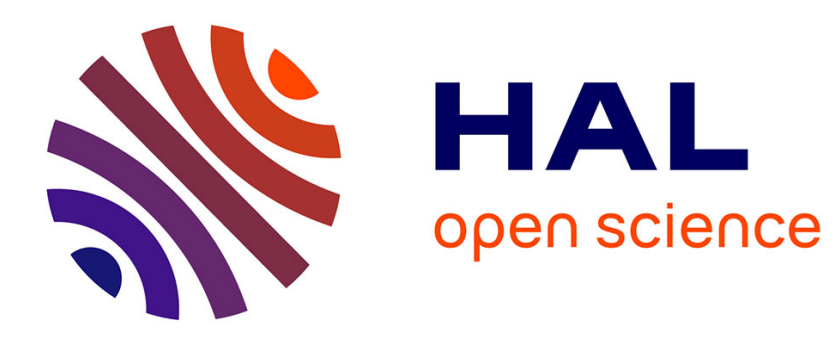

\title{
Mesure de la photoconduction stationnaire dans le sélénium liquide
}

\author{
J. Rabit, J.C. Perron
}

\section{To cite this version:}

J. Rabit, J.C. Perron. Mesure de la photoconduction stationnaire dans le sélénium liquide. Revue de Physique Appliquée, 1982, 17 (5), pp.277-284. 10.1051/rphysap:01982001705027700 . jpa-00244997

\section{HAL Id: jpa-00244997 https://hal.science/jpa-00244997}

Submitted on 1 Jan 1982

HAL is a multi-disciplinary open access archive for the deposit and dissemination of scientific research documents, whether they are published or not. The documents may come from teaching and research institutions in France or abroad, or from public or private research centers.
L'archive ouverte pluridisciplinaire HAL, est destinée au dépôt et à la diffusion de documents scientifiques de niveau recherche, publiés ou non, émanant des établissements d'enseignement et de recherche français ou étrangers, des laboratoires publics ou privés. 
Classification

Physics Abstracts

72.40

\title{
Mesure de la photoconduction stationnaire dans le sélénium liquide
}

\author{
J. Rabit et J. C. Perron \\ Laboratoire de Génie Electrique de Paris (*), Ecole Supérieure d'Electricité, \\ Plateau du Moulon, 91190 Gif sur Yvette, France
}

(Reçu le 14 septembre 1981, révisé le 8 janvier 1982, accepté le 20 janvier 1982)

\begin{abstract}
Résumé. - Après une brève description des techniques utilisées, nous donnons des résultats des mesures du courant de photoconduction du sélénium liquide dans le domaine suivant : température $220^{\circ} \mathrm{C}-350^{\circ} \mathrm{C}$, énergie photonique $1,5 \mathrm{eV}-3 \mathrm{eV}$, champ électrique $100 \mathrm{~V} . \mathrm{cm}^{-1}-2500 \mathrm{~V} . \mathrm{cm}^{-1}$. Nous avons observé un effet bolométrique qui est négligeable dans les conditions expérimentales retenues et un phénomène transitoire lent que nous attribuons à la migration d'ions $\mathrm{O}^{--}$et $\mathrm{Na}^{+}$. Nous déterminons une bande interdite de photoconduction de $2 \mathrm{eV}$ ainsi que ses variations avec la température. Enfin nous associons le temps de vie des porteurs à la densité des bouts de chaînes.
\end{abstract}

Abstract. - The used techniques are first described. Then some results on the measurements of photocurrent in liquid selenium are reported for : $220^{\circ} \mathrm{C} \leqslant T \leqslant 350^{\circ} \mathrm{C}, 1.5 \mathrm{eV} \leqslant h v \leqslant 3 \mathrm{eV}$ and

$$
100 \mathrm{~V} . \mathrm{cm}^{-1} \leqslant E \leqslant 2500 \mathrm{~V} . \mathrm{cm}^{-1} \text {. }
$$

A heating phenomenon has been observed but it is negligeable in our experiments. A slow time varying phenomenon is attributed to the migration of $\mathrm{O}^{2-}$ and $\mathrm{Na}^{+}$ions. A photoconductivity gap closed to $2 \mathrm{eV}$ and its temperature variations are obtained. Endly the hole life time is associated with the density of chain ends.

1. Introduction. - Les nombreuses études effectuées ces dernières années concernant les matériaux amorphes ont, entre autres choses, permis de dissocier l'existence de propriétés semiconductrices de la présence d'un ordre à longue distance et, ainsi, de mettre en évidence l'importance de l'ordre local. Il n'est donc pas surprenant que des éléments comme le soufre ou le sélénium qui conservent une structure chaînée au-delà du point de fusion manifestent un comportement semiconducteur à l'état liquide. Dans le cas du sélénium fondu on dispose de quelques résultats relatifs aux propriétés structurales [1] [2] [3], mais, ceux traitant des caractéristiques électroniques sont rares en dehors de quelques mesures de conductivité, d'absorption optique et de résonance de spin électronique [4] [5] [6] [7].

L'étude de la photoconduction est un outil privilégié permettant l'analyse des défauts de structure. Il est très utilisé dans le cas des semiconducteurs cristallins ou amorphes et, dans cet article, nous décrivons les techniques expérimentales que nous avons étudiées et mises en œuvre afin de pouvoir mesurer la photoconduction dans la phase liquide du sélénium, mesures qui posent des problèmes spéci-

$\left(^{*}\right)$ Associé au C.N.R.S. fiques. Nous explicitons ensuite notre expérimentation en régime stationnaire et pulsé puis nous disons quelques mots des résultats obtenus et fournissons les éléments d'une interprétation phénoménologique.

2. Techniques expérimentales. - Deux structures géométriques sont ordinairement mises en œuvre pour l'étude de la photoconduction, l'une dite « transversale » dans laquelle le flux lumineux est orthogonal au champ électrique l'autre "longitudinale" dans laquelle ces deux grandeurs sont parallèles (Fig. 1). Les dimensions des cellules sont importantes par rapport à deux grandeurs physiques : la longueur d'absorption et le libre parcours moyen des porteurs excités. Un échantillon est ainsi considéré comme mince ou épais selon que sa dimension parallèle à la direction d'incidence du flux lumineux est faible ou grande devant la distance d'absorption. Il est massif si la distance interélectrode est grande devant le libre parcours moyen des porteurs et si les phénomènes de surface sont négligeables. Les fortes valeurs de l'absorption optique du sélénium liquide pour les longueurs d'onde utilisées et l'impossibilité qu'il y a à réaliser des couches liquides suffisamment minces font que nos échantillons sont toujours épais et massifs.

La lumière incidente fournit la seconde caractéris- 


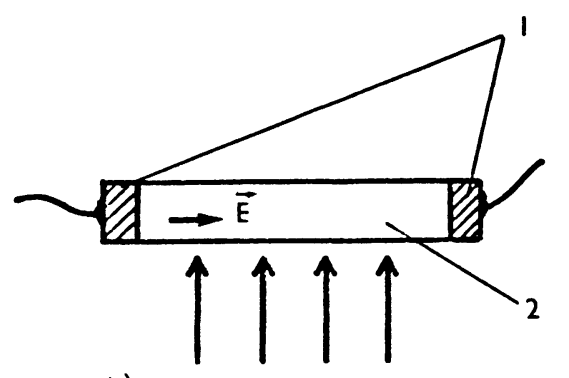

A)

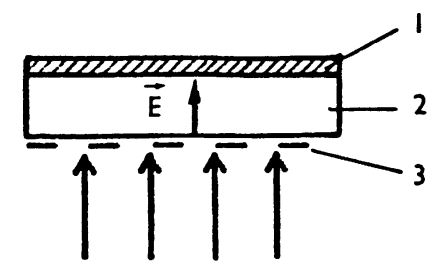

B )

Fig. 1. - Géométries : A) Transversale; B) Longitudinale. 1. Electrodes massives; 2. Matériau; 3. Electrode semitransparente.

[Structures : A) Transverse; B) Longitudinal. 1. Bulky electrodes; 2. Material; 3. Semi transparent electrode.]

tique des expériences de photoconduction. Généralement monochromatique deux cas se présentent : si l'intensité est constante ou modulée avec une période grande devant le temps de réponse du matériau le régime est stationnaire dans le cas contraire ou avec une excitation lumineuse impulsionnelle on observe un régime transitoire.

Nos mesures ont été conduites en mettant en œuvre d'une part des sources à spectre large (lampe à décharge dans le xénon en régime continu ou impulsionnel) d'autre part une source à spectre étroit (laser continu à argon ionisé de puissance lumineuse égale à 4 watts en émission multi-raies). La modulation temporelle est obtenue par hachage mécanique pour observer le régime stationnaire (fréquence inférieure à $10 \mathrm{kHz}$ ) par modulation acousto-optique du faisceau laser monochromatique pour l'étude des régimes transitoires (des impulsions lumineuses aussi brèves que 20 ns sont ainsi obtenues). Les difficultés expérimentales sont essentiellement dues à deux facteurs, d'une part la grande activité chimique du sélénium à l'état liquide d'autre part la faible amplitude des signaux à mesurer avec des impédances de source importantes. Ce dernier problème a été résolu par l'utilisation d'un voltmètre à détection synchrone dans le cas des régimes stationnaires et celle d'un échantillonneur moyenneur pour l'analyse des régimes transitoires.

La forte activité chimique des chalcogénures à l'état liquide conduit à certaines difficultés dans la réalisation des cellules de mesure. Ainsi, seuls le carbone et certains métaux réfractaires sont susceptibles d'être utilisés comme électrodes.

Parmi les métaux, seul le tungstène offre une sta- bilité suffisante dans la mesure où la température reste inférieure à $450^{\circ} \mathrm{C}$. Le sélénium liquide est structuralement constitué de chaînes longues $\left(>10^{4}\right.$ atomes par chaîne) et, de ce fait, sa viscosité est importante [8]. Cette propriété, jointe à la faible valeur de la pression de vapeur, nous permet d'obtenir une étanchéité suffisante avec un contact verre sur verre. Seul un polyimide (kapton) est assez stable chimiquement et thermiquement pour la réalisation des joints et séparateurs dans les cellules destinées aux mesures en géométrie longitudinale.

Ainsi en géométrie transversale, nous avons utilisé des cellules en verre optique ou en quartz composées d'une embase carrée sur laquelle un anneau est soudé par diffusion moléculaire. Les électrodes, d'abord en carbone massif, ont finalement été réalisées par pulvérisation cathodique de tungstène (Fig. 2). Le remplissage est assuré par une pastille de matériau préformé. Pour cela, on prépare un lingot cylindrique par fusion sous vide dans un tube scellé. Ce cylindre est ensuite scié en rondelles que l'on polit et nettoie avant de les placer sous atmosphère d'argon dans la cellule.

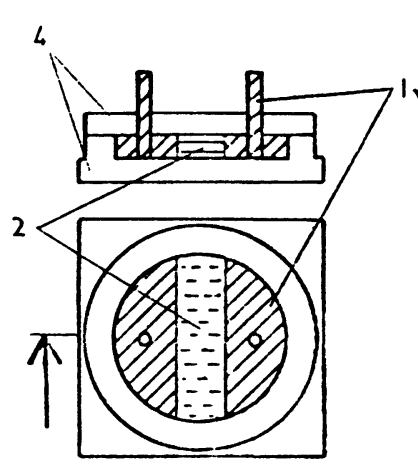

A)

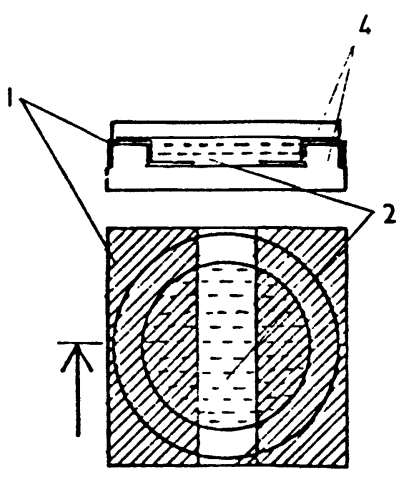

B)

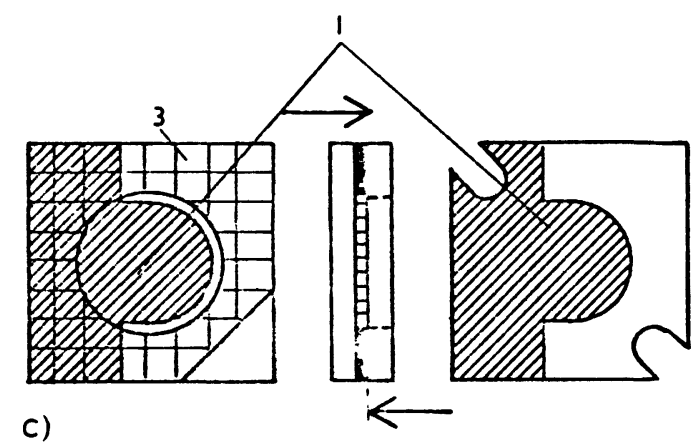

Fig. 2. - Cellules : A) Epaisses à électrodes de carbone; B) Epaisses à électrodes de tungstène pulvérisé ; C) Mince à électrodes d'oxyde d'étain. 1. Electrode; 2. Sélénium; 3. Kapton; 4. Verre/Quartz.

[Cells : A) Thick cell with carbon electrodes; B) Thick cell sputtered tungsten electrodes; C) Thin cell with tin oxide electrodes. 1. Electrode; 2. Selenium; 3. Kapton.]

En structure longitudinale on utilise deux plaques de verre sur lesquelles un dépôt semi-transparent et conducteur d'oxyde d'étain joue le rôle d'électrode. 
La séparation $(10 \mu \mathrm{m}, 25 \mu \mathrm{m}, 50 \mu \mathrm{m})$ est assuré par un joint en kapton (Fig. $2 b$ ). Le matériau est déposé par évaporation sous vide et son épaisseur est déterminée par interférométrie infra-rouge. Ces échantillons n'ont pas une durée de vie très longue (quelques heures) mais permettent néanmoins de faire des mesures.

La mise en température est assurée par un four tubulaire à axe vertical présentant une fenêtre transparente dans sa partie inférieure. De façon à pouvoir contrôler l'atmosphère interne ce four est étanche (Fig. 3). La régulation est obtenue par une chaîne asservie de type P.I.D. commandant la puissance de chauffage par thyristors; une chemise d'eau assure le refroidissement. L'utilisation d'un bobinage antiinductif et d'une commande "en train d'onde" a permis de réduire le niveau de parasites à une valeur

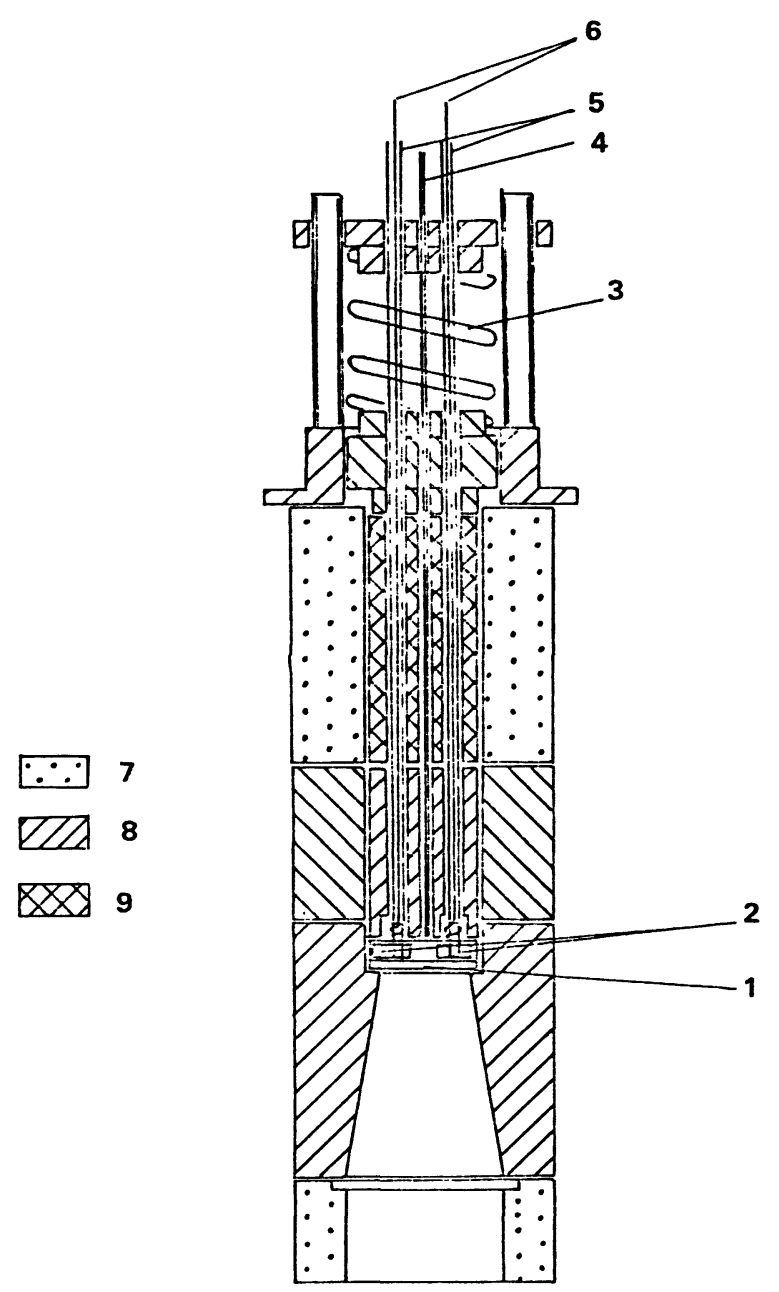

Fig. 3. - Four intérieur : 1. Cellule; 2. Electrodes; 3. Ressort ; 4. Thermocouple de mesure; 5. Gaines isolantes en alumine; 6 . Amenées courant à l'échantillon; 7. Steatite; 8 . Acier inoxydable; 9 . Alumine.

[Inner furnace : 1. Cell ; 2. Electrodes; 3. Spring; 4. Measurement thermocouple; 5. Alumina insulating sheath; 6. Wires to sample; 7. Steatite; 8. Inox steel; 9. Alumina.] compatible avec les possibilités de mesures. L'intérieur comportant la cellule est présenté sur la figure 3.

3. Résultats. - 3.1 GÉNÉRALITÉs. - L'appareillage que nous avons décrit et les propriétés physiques du sélénium ont limité le domaine d'étude de la photoconduction. Ainsi au voisinage de sa température de fusion le sélénium pur $\left(218^{\circ} \mathrm{C}\right)$ présente une résistivité qui, selon les auteurs, c'est-à-dire selon la nature des impuretés varie de $10^{6}$ à $10^{7} \Omega$. cm [5] [9][10]. Le phénomène de photoconduction est très sensible aux impuretés et les variations, en valeurs absolues, des signaux observés selon les échantillons nous obligent à donner les résultats correspondants en unités arbitraires. En géométrie transversale la résistance de l'échantillon est alors voisine de $100 \mathrm{M} \Omega$ et décroît rapidement avec la température car la conductivité électrique est thermiquement activée (l'énergie d'activation est comprise entre 0,5 et $1 \mathrm{eV}$ ). La diminution de la résistance d'échantillon ne rend pas les mesures plus aisées en raison des effets de contacts et des instabilités dans le liquide. La diminution de la durée de vie des porteurs avec l'augmentation de la température s'ajoutant à cela fait que les mesures ne sont pratiquement plus possibles à des températures supérieures à $350^{\circ} \mathrm{C}$.

Les phénomènes de non-linéarité et de commutation dans le sélénium liquide pur [11] [12] limitent le champ électrique applicable à $2500 \mathrm{~V} \cdot \mathrm{cm}^{-1}$ environ et en géométrie transversale, la tension maximale admissible sur l'étage d'entrée de l'amplificateur de mesure nous interdit l'utilisation de champs électriques supérieurs à $250 \mathrm{~V} . \mathrm{cm}^{-1}$. Enfin, pour rendre l'effet thermique négligeable nous limitons le flux de photons incidents à $10^{17} \mathrm{ph} . \mathrm{cm}^{-2} \mathrm{~s}^{-1}$ en régime stationnaire. L'usage d'une lampe à décharge dans le xénon limite la valeur de l'énergie $h v$ d'un photon à $3 \mathrm{eV}$ pour les valeurs supérieures et la diminution du signal empêche les mesures pour des énergies inférieures à $1,5 \mathrm{eV}$. Ce spectre est néanmoins bien adapté au cas du sélénium pur. En résumé, nos mesures de photoconduction se situent dans le domaine suivánt :

$$
\begin{aligned}
220 & <T<350^{\circ} \mathrm{C} \\
1,5 & <h v<3 \mathrm{eV} \\
100 & <F<2500 \mathrm{~V} . \mathrm{cm}^{-1} .
\end{aligned}
$$

3.2 EFFET D'ÉleCtrodes. - En géométrie transversale les deux électrodes étant constituées de tungstène, on peut normalement supposer le système symétrique. L'inversion de la tension appliquée nous a cependant mis en évidence une transition lente (quelques minutes) entre les deux états stationnaires symétriques. Après inversion, le courant moyen traversant l'échantillon croît, passe par un maximum et diminue pour retrouver sa valeur initiale changée de signe. Le courant de photoconduction quant à lui commence par diminuer, passe par un minimum, puis par un maximum nettement supérieur à sa valeur 
initiale qu'il retrouve au terme d'une lente diminution (Fig. 4). Le temps de passage par le maximum du courant ne dépend que de la température et est indépendant du matériau constitutif des électrodes. Par contre les amplitudes de variations sont nettement diminuées par une préparation de l'échantillon de sélénium sous atmosphère d'argon. Nous interprétons ces phénomènes comme traduisant le transit d'impuretés ionisées d'une électrode à l'autre. L'estimation de la mobilité à partir de ces mesures conduit à des valeurs qui sont habituelles pour des ions dans un liquide. C'est-à-dire pour les ions oxygène $\mathrm{O}^{--}$ $1,7 \times 10^{-5} \mathrm{~cm}^{2} \mathrm{~V}^{-1} \mathrm{~s}^{-1}$ et pour les ions sodium $\mathrm{Na}^{+}$ $3,5 \times 10^{-5} \mathrm{~cm}^{2} \mathrm{~V}^{-1} \mathrm{~s}^{-1}$.

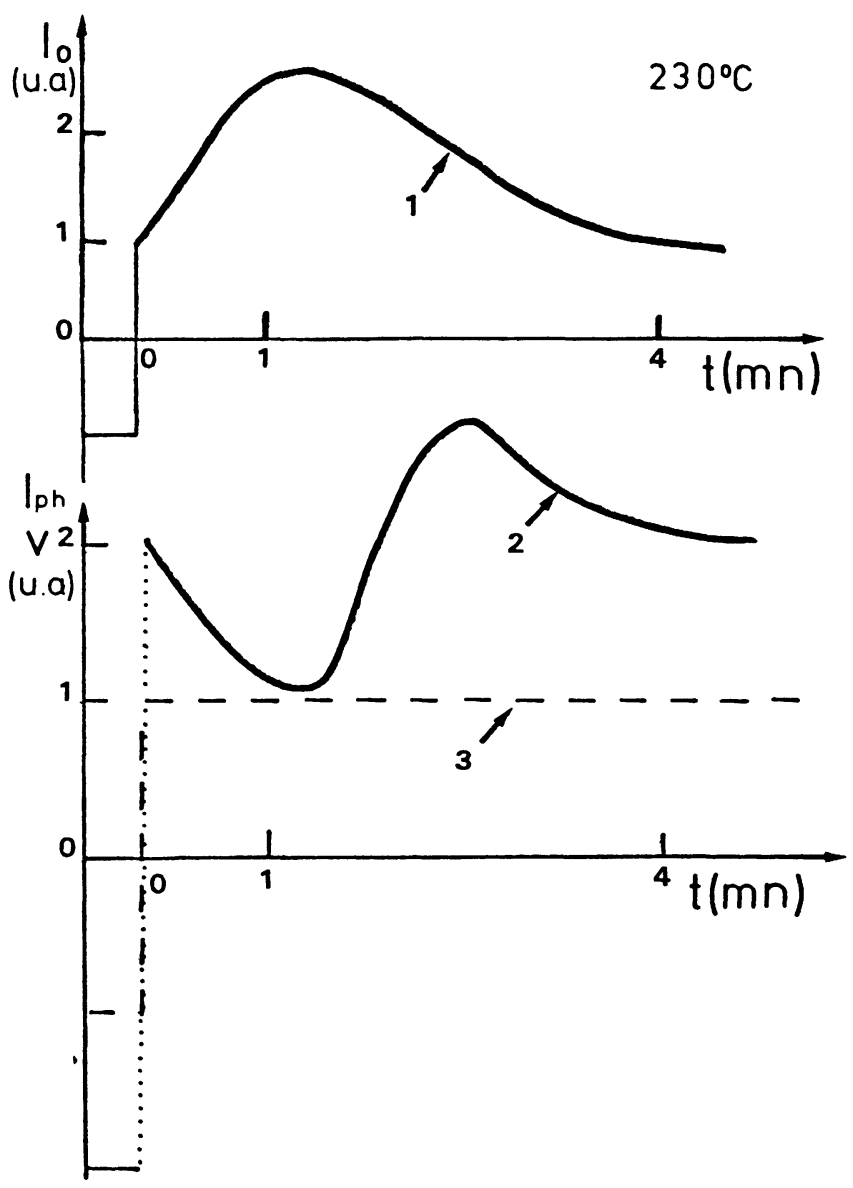

Fig. 4. - Effets de contacts (géométrie transversale) : 1. Courant d'obscurité; 2. Courant de photoconduction; 3. Tension appliquée.

[Contact phenomena : 1. Dark current ; 2. Photoconductivity current ; 3. Applied voltage.]

Ces valeurs ne doivent pas être associées à la viscosité élevée du sélénium, ces mobilités sont, en effet, gouvernées par la structure locale de ce matériau qui présente un volume libre important alors que la viscosité est liée, pour l'essentiel, à la longueur des chaînes constituant le sélénium.

3.3 INFLUENCE D'UN EFFET BOLOMÉTRIQUE. - La conductivité du sélénium liquide est activée thermique- ment et donc, très sensible à une variation de température qui peut être due à l'absorption de lumière. Il nous faut donc évaluer l'importance possible de cet effet thermique de façon à comparer le signal de photoconduction au signal thermique parasite. Dans le cas d'un échantillon mince soumis à un éclairement périodique, l'amplitude de variation de température est donnée par l'expression [13] :

$$
\Delta T=\frac{P_{\mathrm{L}}}{C_{\mathrm{th}}}\left[\left(\frac{K_{\mathrm{th}}}{C_{\mathrm{th}}}\right)^{2}+4 \pi^{2} f^{2}\right]^{-1 / 2}
$$

où $P_{\mathrm{L}}$ est la puissance crête du faisceau lumineux, $f$ la fréquence de hachage, $C_{\mathrm{th}}$ et $K_{\mathrm{th}}$ représentent respectivement la capacité et la conductance thermique de l'échantillon. Ceci permet d'évaluer une borne supérieure du signal d'origine thermique à savoir :

$$
s_{\mathrm{th}}=\frac{V}{4} \cdot \frac{E_{\mathrm{a}}}{k T^{2}} \cdot \Delta T
$$

où $V$ est la tension appliquée et $E_{\mathrm{a}}$ l'énergie d'activation de la conductivité. Avec des valeurs raisonnables pour toutes les quantités impliquées le signal d'origine thermique est égal à $8 \times 10^{-2} \mu \mathrm{V}$ et $0,5 \mu \mathrm{V}$ respectivement en géométrie transversale et longitudinale. Ces valeurs sont surestimées largement dans notre cas ; et elles sont inférieures à la précision des mesures en géométrie transversale et inférieures à $3 \%$ du signal mesuré en géométrie longitudinale. Nous avons également analysé l'influence de la déformation thermique sur un signal carré de photoconduction en comparant les valeurs respectives du fondamental dans chacun

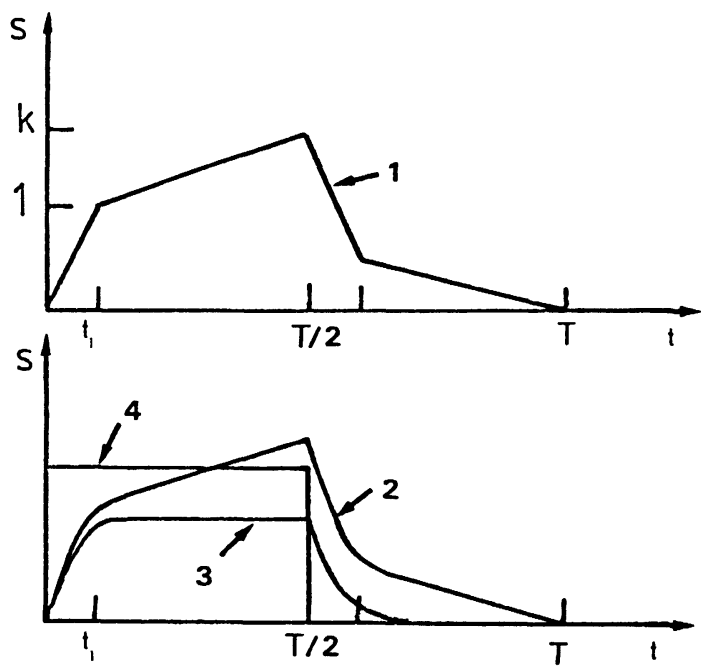

Fig. 5. - Effet thermique : 1. Approximation du signal; 2. Signal réel présentant un effet thermique; 3 . Signal réel sans effet thermique; 4. Intensité lumineuse. Tableau I : Rapport entre les fondamentaux d'un signal déformé et d'un signal pur.

[Bolometric effect : 1. Signal approximation; 2. Actual signal showing heating effect; 3. Actual signal without heating effect; 4. Light intensity. Table I : Ratio between the fundamentals of a heat deformed signal and a pure 'photoconductive signal.] 
des cas. En effet l'utilisation d'une détection synchrone ne permet de mesurer que cette composante. Une erreur de $5 \%$ implique un signal vraiment très déformé (Fig. 5, tableau I).

\begin{tabular}{|l|l|l|l|l|}
\hline$t_{1} k$ & 1 & 1,2 & 1,5 & 2 \\
\hline 0 & 1 & 1,01 & 1,05 & 1,19 \\
\hline 0,1 & 1,00 & 1,01 & 1,06 & 1,22 \\
\hline 0,3 & 0,96 & 0,98 & 1,04 & 1,26 \\
\hline 0,5 & 0,90 & 0,92 & 1,03 & 1,27 \\
\hline
\end{tabular}

Enfin nous avons mené une étude expérimentale qui nous a permis, d'une part de mettre en évidence un effet thermique à basse fréquence et, à haute fré- . quence, un effet de filtrage lié à l'appareillage. Nous avons donc choisi une fréquence de travail $(556 \mathrm{~Hz})$ qui permet de supprimer l'effet bolométrique, de ne pas tenir compte des fréquences de coupures propres à l'instrumentation et de minimiser les constantes de temps de mesure (Fig. 6).

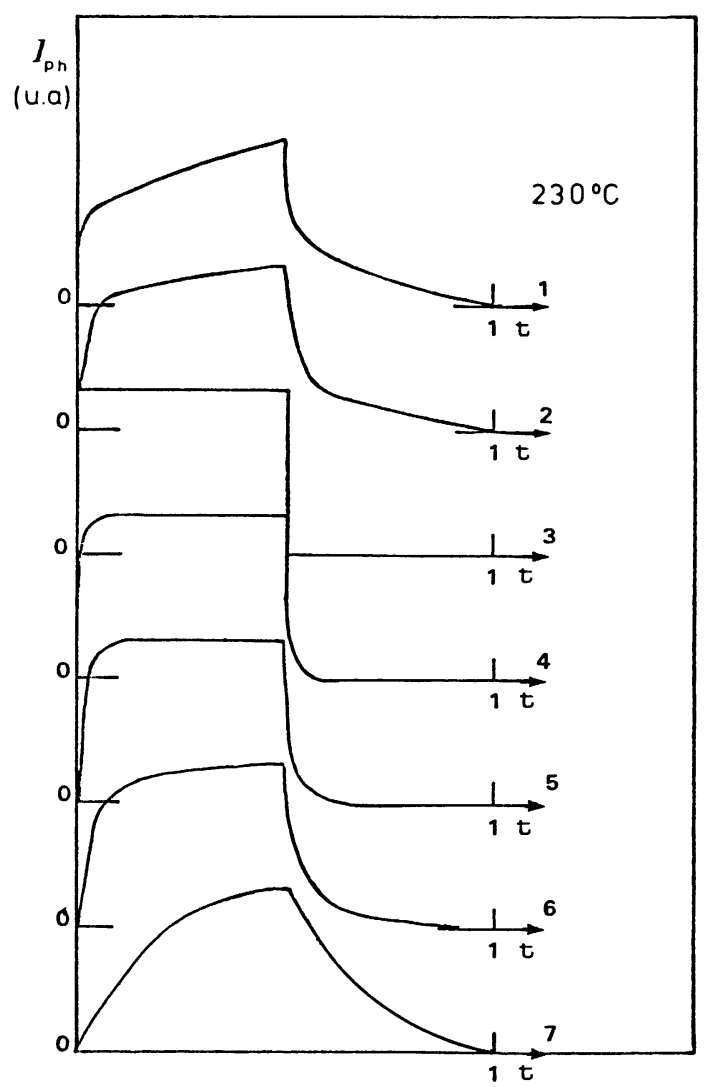

Fig. 6. - Effet thermique (expérimental) : $1.22 \mathrm{~Hz} ; 2$. $71 \mathrm{~Hz}$; 3. $278 \mathrm{~Hz}$; 4. $556 \mathrm{~Hz}$; 5. $1330 \mathrm{~Hz}$; 6. $5150 \mathrm{~Hz}$; 7. $35400 \mathrm{~Hz}$. (Echelle $1=f^{-1}$.)

[Bolometric effect (experimental) : 1. $22 \mathrm{~Hz} ; 2.71 \mathrm{~Hz}$; 3. $278 \mathrm{~Hz}$; 4. $556 \mathrm{~Hz}$; 5. $1330 \mathrm{~Hz}$; 6. $5150 \mathrm{~Hz} ; 7$. $35400 \mathrm{~Hz}$.]
3.4 VARIATIONS DE LA PHOTOCONDUCTION. - Avec le champ électrique. - Dans le sélénium liquide, il apparaît à champ élevé $\left(>3 \mathrm{kV} \mathrm{cm}{ }^{-1}\right)$ des phénomènes de commutation qui interdisent toute mesure de photoconduction. Nos résultats (Fig. 7) montrent une variation linéaire du photocourant et du courant d'obscurité dans tout le domaine de champ électrique et de température accessible. Ceci exclut tout phénomène de charge d'espace et de variation de la mobilité des porteurs avec le champ électrique.

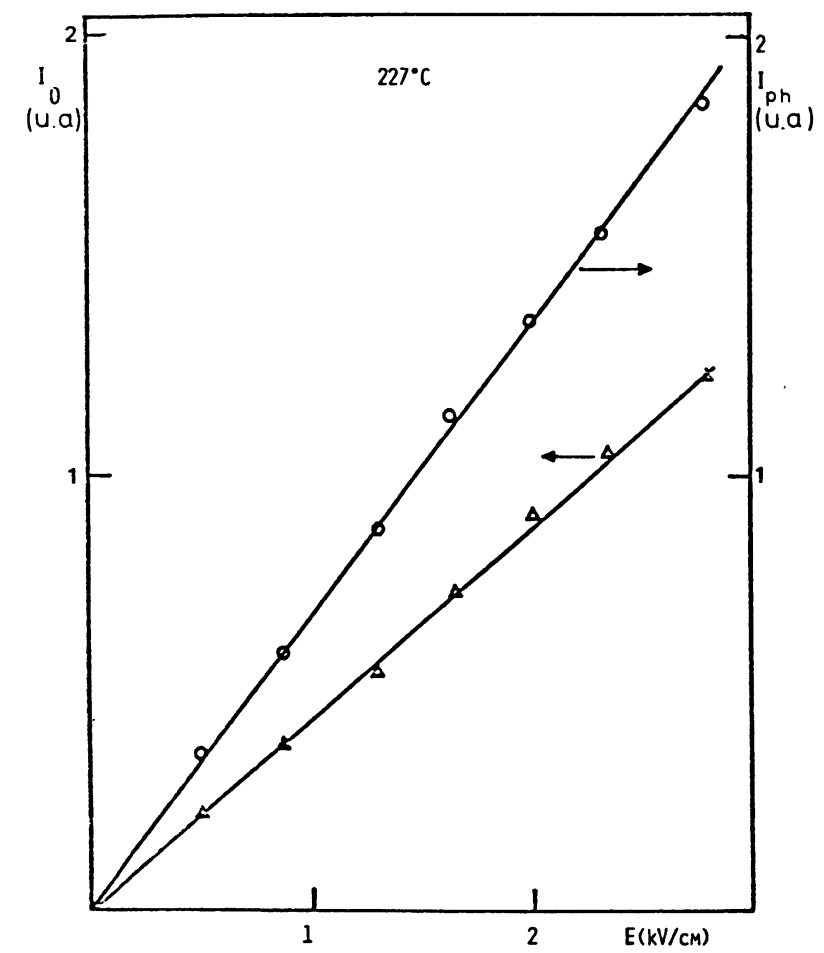

Fig. 7. - Courant d'obscurité $\left(I_{0}\right)$ et courant de photoconduction $\left(I_{\mathrm{ph}}\right)$ en fonction du champ électrique $(E)$.

[Dark current $\left(I_{0}\right)$ and photoconductivity current $\left(I_{\mathrm{ph}}\right)$ versus electric field $(E)$.]

- Avec le flux de photons. - L'utilisation d'un laser continu dè puissance a permis d'étendre la plage de détection et de mesure du courant de photoconduction jusqu'à $390^{\circ} \mathrm{C}$ et à des flux lumineux importants $\left(10^{18}\right.$ photons $\left.\mathrm{cm}^{-2} \mathrm{~s}^{-1}\right)$. Un comportement linéaire a été observé dans tous les cas et quelle que soit la longueur d'onde de la lumière utilisée (Fig. 8). Aussi le processus de recombinaison ne fait-il, probablement, intervenir qu'un seul groupe de centres recombinants.

- Avec l'énergie des photons. - Si l'épaisseur de l'échantillon est telle que toute la lumière soit absorbée alors en géométrie transversale comme en géométrie longitudinale, la variation de la conductance de l'échantillon peut s'écrire :

$$
\Delta \sigma=k \cdot I_{\lambda} \cdot \mu \cdot \tau \cdot \eta
$$

$I_{\lambda}$ est le flux lumineux incident, $\mu$ la mobilité des porteurs, $\tau$ leur durée de vie et $\eta$ le rendement quantique. 


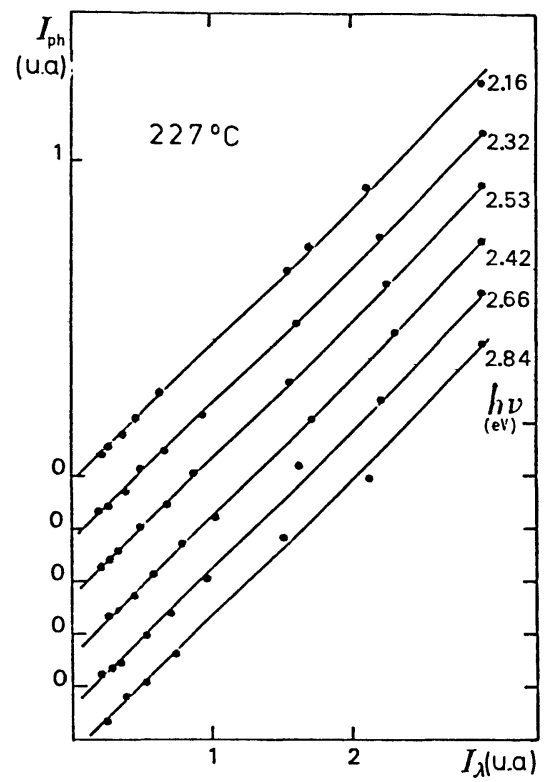

Fig. 8. - Courant de photoconduction en fonction de l'intensité lumineuse.

[Photoconductivity current versus light intensity.]

Cette relation n'est valable que si $\Delta \sigma$ est très inférieur à $\sigma$. Cette condition est toujours vérifiée dans nos expériences, donc le courant de photoconduction est indépendant de la valeur de l'absorption optique. Les variations de ce courant avec l'énergie des photons représentées sur la figure 9 traduisent alors essentiellement l'évolution du rendement quantique $\eta$ avec la longueur d'onde.

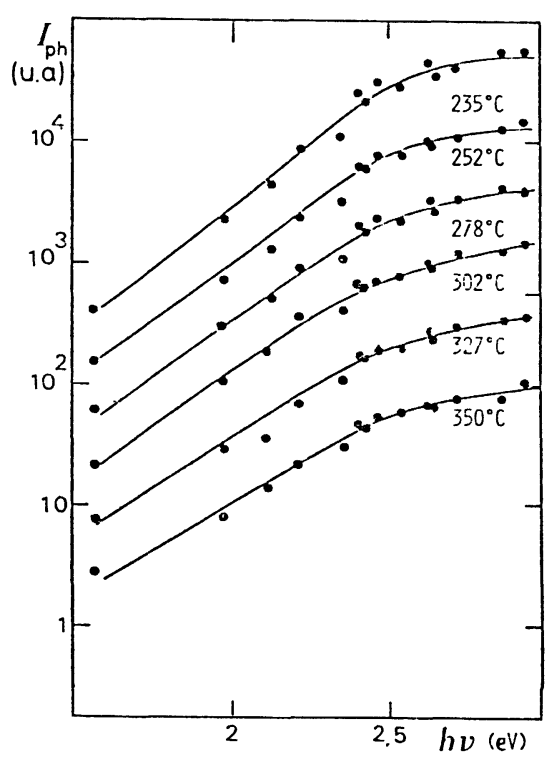

Fig. 9. - Courant de photoconduction $\left(I_{\mathrm{ph}}\right)$ en fonction de l'énergie des photons à tension appliquée constante.

[Photoconductivity current versus photon energy.]

- Avec la fréquence. - La fréquence de hachage du flux lumineux n'a pas d'influence sur le courant de photoconduction sauf à très basse fréquence $(<40 \mathrm{~Hz})$ où apparaît un effet thermique et à haute fréquence en raison de l'appareillage de mesure. La seule conclusion que l'on puisse en tirer est que le temps de réponse du photoconducteur étudié est inférieur à $10 \mu \mathrm{s}$.

- Avec la température. - Le tracé du logarithme du courant de photoconduction en fonction de l'inverse de la température met en évidence que l'inverse du photocourant est activé thermiquement. La détermination de l'énergie d'activation conduit à une valeur qui varie avec l'énergie des photons incidents (Fig. 10).

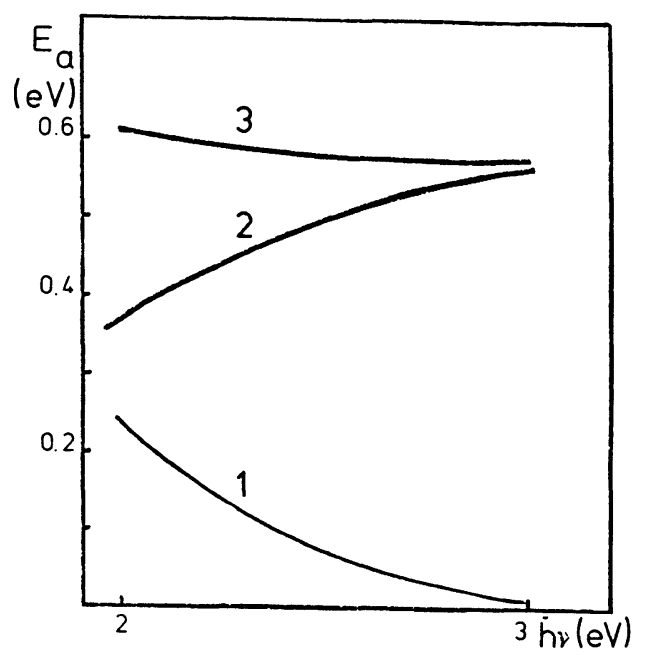

Fig. 10. - Energies d'activation du : 1. Rendement quantique ; 2 . Courant de photoconduction ; 3 . Temps de vie des trous.

[Activation energy : 1. Quantum efficiency; 2. Photoconductivity current ; 3 . Hole life-time.]

4. Discussion. - En géométrie longitudinale nous observons un courant de photoconduction uniquement quand l'électrode éclairée est polarisée positivement. La conductivité électrique est donc essentiellement assurée par des trous, puisque les porteurs de charge opposée à la polarité de l'électrode, au voisinage de laquelle ils sont créés en raison de la grande valeur du coefficient d'absorption optique, se recombinent quasi immédiatement sur celle-ci. Ceci est conforme aux résultats généralement obtenus dans les chalcogénures et, dans le cas du sélénium liquide au signe du pouvoir thermoélectrique. Les variations du photocourant en fonction de l'énergie des photons nous ont permis d'extrapoler linéairement une bande interdite de photoconduction. Cette méthode a été choisie de façon empirique comme donnant les résultats les plus reproductibles. Ses variations avec la température mettent en évidence un coefficient de température moyen égal à $9 \times 10^{-4} \mathrm{eV} \cdot \mathrm{K}^{-1}$ (Fig. 11). Cette valeur est d'un ordre de grandeur supérieure à celle observée dans le solide mais est voisine de celle obtenue en phase liquide [14]. L'évolution structurale 
rapide du liquide au voisinage du point de fusion justifie la décroissance plus marquée de cette bande interdite aux basses températures [15].

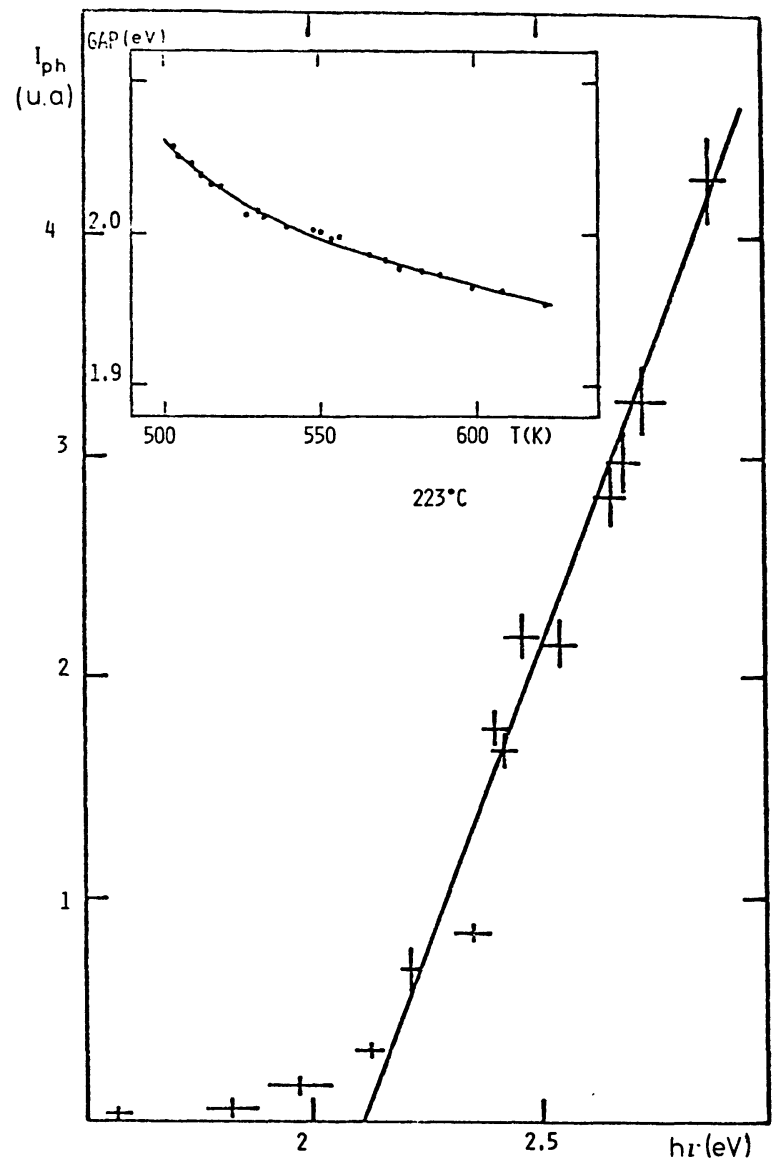

Fig. 11. - Extrapolation du gap de photoconduction. Encart : Variations de ce gap avec la température.

[Photoconductivity gap extrapolation. Inset : temperature dependance of the gap.]

Les variations linéaires avec le champ électrique et l'intensité lumineuse incidente montrent d'une part qu'il n'y a pas de phénomène de saturation et d'autre part que le retour à l'équilibre des porteurs en excès peut s'analyser par un seul groupe de centres recombinants. Enfin, le courant de photoconduction peut s'exprimer par :

$$
i_{\mathrm{ph}}=K \cdot \mu \cdot \tau \cdot \eta
$$

La conduction étant assurée par des mouvements diffusifs des porteurs, la mobilité n'est pas activée et, de ce fait, peut être considérée comme variant peu avec la température par rapport à $i_{\mathrm{ph}}, \tau$ et $\eta$ qui sont activées. Utilisant alors l'énergie d'activation du rendement quantique trouvée dans le cas de la phase amorphe [16] considérée comme un état figé de la phase liquide on peut écrire :

$$
E_{\mathrm{ph}}=E_{\tau}+E_{\eta}
$$

où $E_{\mathrm{ph}}, E_{\tau}$ et $E_{\eta}$ sont les énergies d'activation respectives de la phutoconductivité, du temps de vie des trous et du rendement quantique. $E_{\mathrm{ph}}$ nous est donnée par les courbes de la figure 10 et nous pouvons alors déterminer $E_{\tau} . E_{\tau}$ est trouvée égale à $0,57 \mathrm{eV}$ et indépendant de la longueur d'onde. Ce résultat est physiquement satisfaisant car un trou une fois créé et thermalisé ne conserve pas la "mémoire » de l'énergie du photon qui l'a créé. La valeur de cette énergie d'activation peut alors suggérer d'associer les centres recombinants à des extrémités de chaînes. En effet le nombre de bouts de chaînes dans le sélénium liquide est activé thermiquement. Les mesures de viscosité [8] et de résonance électronique de spin [6] fournissent en effet respectivement des énergies d'activation de $0,5 \mathrm{eV}$ et $0,63 \mathrm{eV}$.

5. Conclusion. - Nous avons donc décrit ici les techniques expérimentales que nous avons mis en œuvre dans nos mesures de photoconduction dans le sélénium liquide. Les difficultés importantes rencontrées ont été résolues aux températures inférieures à $350^{\circ} \mathrm{C}$. Après une analyse rapide de l'effet thermique nous rendons compte de phénomènes transitoires lents que nous attribuons à des migrations d'ions. Enfin nous présentons des résultats originaux de photoconduction en régime stationnaire. L'étude est menée en fonction de divers paramètres et permet la détermination d'un gap de photoconductivité et de l'énergie d'activation du temps de vie des trous que nous comparons aux valeurs obtenues par d'autres techniques. Cette voie semble fructueuse pour l'analyse des phénomènes de transport électronique dans les semiconducteurs liquides. Cette étude peut permettre une meilleure connaissance de la phase amorphe du sélénium dont l'importance pratique n'est plus à démontrer.

\section{Bibliographie}

[1] Long, M., Gallison, P., Alben, R., Connell, G. A. N., Phys. Rev. B 13 (1976) 1821.

[2] Misawa, M., Suzuki, K., J. Phys. Soc. Japan 44 (1978) 1612.

[3] Malaurent, J. C., Dixmier, J., Proc. 8th Int. Conf. Am. Liq. Semiconductors Boston (1979) p. 1227.
[4] Rabit, J., Perron, J. C., Phys. Status Solidi (b) 65 (1974) 255.

[5] Mac Millan, P. W., Shutov, S. D., J. Non-Crystallogr. Solids 24 (1977) 307.

[6] Koningsberger, D. C., Van Wolput, J. H. M. C., Rieter, P. C. U., Chem. Phys. Lett. 8 (1971) 145. 
[7] Warren, W. J., Dupree, R., Phys. Rev. B 22 (1980) 2257.

[8] Rialland, J. F., Perron, J. C., Revue Phys. Appl. 11 (1976) 263.

[9] Baker, E. H., J. Chem. Soc. A (1967) 1558.

[10] Abdinov, D. Sh., Namazov, V. R., Aliev, G. M., Izv. Akad. Nauk. SSSR Neorg. Mat. 10 (1974) 1960.

[11] Glazov, V. M., Aivazov, A. I., Zelenov, A. V., VADov, G. I., Sov. Phys. Semicond. 10 (1976) 381.
[12] Perron, J. C., J. Non Crystallogr. Solids 8-10(1972) 272.

[13] Moustakas, T. D., Connell, G. A. N., J. Appl. Phys. 47 (1976) 1322.

[14] Motт, N. F., Phil.Mag. 24 (1971) 1.

[15] MotT, N. F., Davis, E. A., In « Electronic Processes in Non-crystalline Materials " (Clarendon Press Oxford) 1971, p. 88.

[16] PAI, D. M., ENCK, R. C., Phys. Rev. B 11 (1975) 5163. 